\title{
Noncomplicated Surgical Removal of Fractured Maxillary Central Incisor from Lower Lip and Its Reattachment: A Case Report
}

\author{
Megha Narayan ${ }^{1}$ Rajat Kumar Singh ${ }^{1} \quad$ Aparna Singh ${ }^{1} \quad$ Siddharth Anand ${ }^{1} \quad$ Sulekha ${ }^{1}$
}

${ }^{1}$ Department of Pedodontics and Preventive Dentistry, Buddha Institute of Dental Sciences and Hospital, Patna, Bihar, India
Address for correspondence Megha Narayan, BDS, Department of Pedodontics and Preventive Dentistry, Buddha Institute of Dental Sciences and Hospital, Patna, Bihar-800026, India (e-mail: meghanarayan007@gmail.com).
Abstract Keywords
- dental trauma
- lower lip
- tooth fragment
- adolescent

\begin{abstract}
Traumatic injuries to maxillary anterior teeth while playing are common in children and adolescents. Sequelae of such trauma include broken, lost, aspirated, and swallowed tooth. At times, the broken tooth fragments get embedded in the adjoining soft tissue and frequently get neglected during diagnosis. The present case report discusses the case of a 13-year-old adolescent who had broken tooth fragment emerged in the lower lip and its surgical excision to prevent detrimental reactions and scarring.
\end{abstract}

\section{Introduction}

Trauma to the teeth and its supporting structures can be caused by falls, vehicle accidents, sports, assaults, etc. Out of all these factors, falls, traffic accidents, and sports activities are the most common etiologic factors. ${ }^{1}$ In dental traumatic injury, crown fracture is the most common. ${ }^{2}$ Incidence of noncomplicated and complicated tooth fractures due to trauma in children has been reported to be 28 to $44 \%$ and 11 to $15 \%$, respectively. ${ }^{3}$

Teeth mainly involved are the maxillary incisors due to their vulnerable position in the arch, proclination, and short upper lip. One major hazard following the dental trauma is the emerged fractured tooth fragments in the surrounding soft tissues, such as buccal mucosa, tongue, and lips, but the most prominent site is the lower lip. If the embedded tooth fragments remain undetected for a longer period of time, it may lead to infection and fibrosis. ${ }^{4-6}$ This article projects the significance of appropriate clinical and radiographic examination of soft and hard tissues and retrieval of embedded fractured tooth fragment from lower lip after trauma and reattachment of broken tooth fragment.

DOI https://doi.org/ $10.1055 / \mathrm{s}-0041-1731101$ ISSN 2321-1482

\section{Case Report}

A healthy 13-year-old girl reported to the Department of Paedriatic and Preventive Dentistry with pain and swelling in the lower lip for the previous 20 days. The patient had sustained trauma 10 months back while she fell in the school playground. Immediately after the trauma, the patient's father took her to the nearby medical practitioner, where she was given primary treatment and referred to a specialist dental practitioner. The soft-tissue lacerations were sutured; analgesics, antibiotics, and tetanus toxoid vaccine were given. On extraoral examination, a scar was present beneath the lower lip, right from the midline ( - Fig. 1). Intraoral examination revealed enamel and dentine fracture of right maxillary incisor ( - Fig. 2). On palpation, a round, hard mass swelling of size $1 \times 1 \mathrm{~cm}$ was felt on the lower lip at the right side of the midline ( $\boldsymbol{- F i g . 3}$ ). As we were suspicious, we advised for an intraoral periapical radiograph of the lower lip along with the maxillary incisors. To our surprise, on radiographic interpretation, it revealed a solitary radiopaque mass similar to the fractured tooth segment ( $\boldsymbol{- \text { Fig. }}$ 4). On pulp vitality test, the fractured maxillary incisor responded vital pulp.

(C) 2021. Bhojia Dental College and Hospital affiliated to Himachal Pradesh University.

This is an open access article published by Thieme under the terms of the Creative Commons Attribution-NonDerivative-NonCommercial-License, permitting copying and reproduction so long as the original work is given appropriate credit. Contents may not be used for commercial purposes, or adapted, remixed, transformed or built upon. (https://creativecommons.org/licenses/by-nc-nd/4.0/).

Thieme Medical and Scientific Publishers Pvt. Ltd. A-12, 2nd Floor, Sector 2, Noida-201301 UP, India 


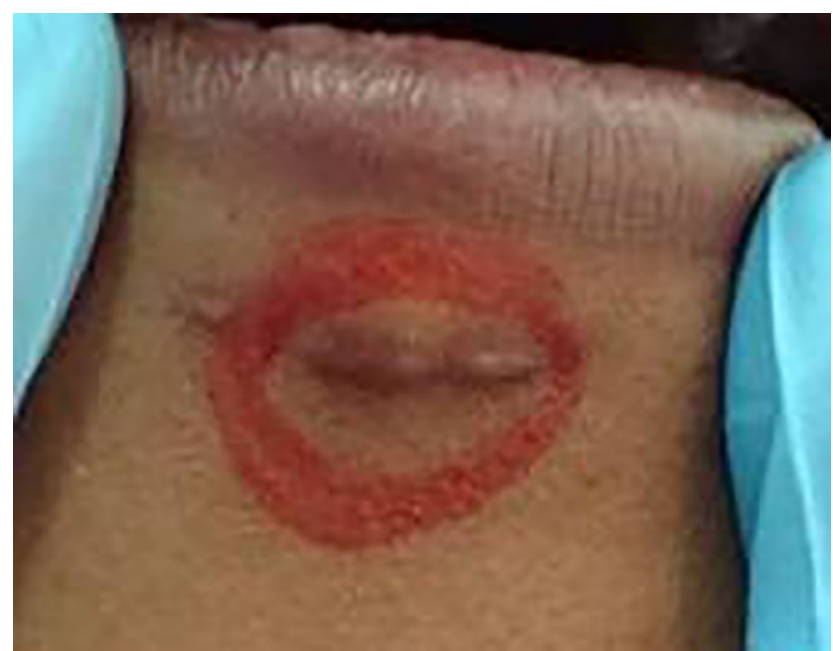

Fig. 1 Preoperative extraoral view of lesion.

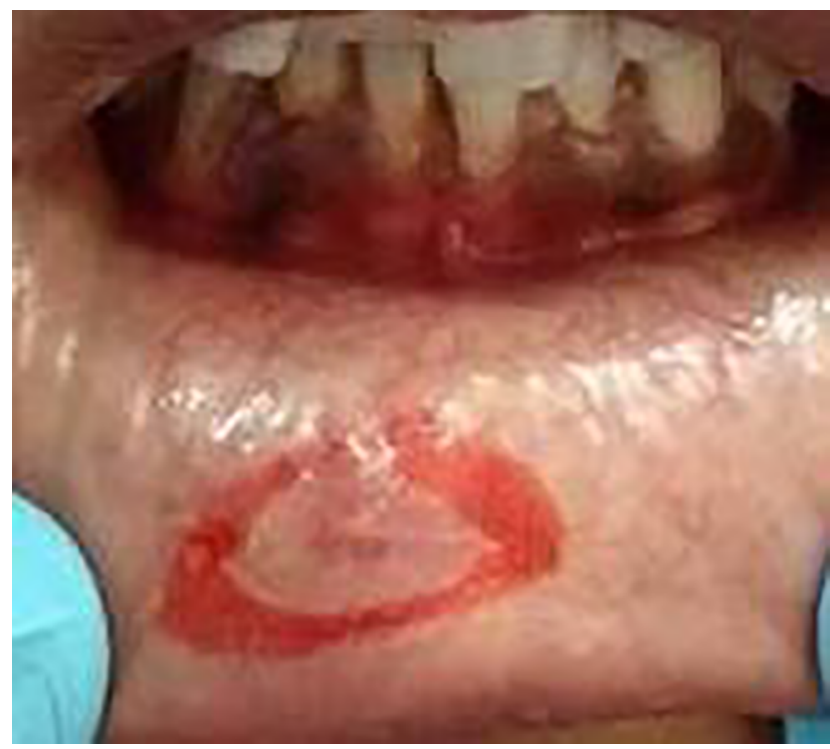

Fig. 3 Preoperative intraoral view of lesion.

Hence, after thorough clinical and radiographic examination, we came to the conclusion that there is an embedded fractured right maxillary central incisor fragment on the right side of the lower lip. Noncomplicated surgical removal of the embedded fractured tooth fragment from the lower lip, followed by its reattachment, was planned. Detailed treatment procedures were explained to the patient as well as to her parents and a written consent for the same was taken.

Under all aseptic conditions, local anesthesia was administered on the right side of the lower lip with a 32-gauze needle. A horizontal incision of $1 \mathrm{~cm}$ was made in the proximity of the hard elevated mass to expose the tooth fragment. The broken fragment of maxillary central incisor was retrieved with the mosquito forceps (-Fig. 5). The area was examined thoroughly and was approximated with 4-0 Vicryl sutures (-Fig. 6). Postoperative instructions were provided and medications were prescribed.

The tooth fragment was cleaned and kept in 10\% formalin in a sterilized container. The patient was recalled after

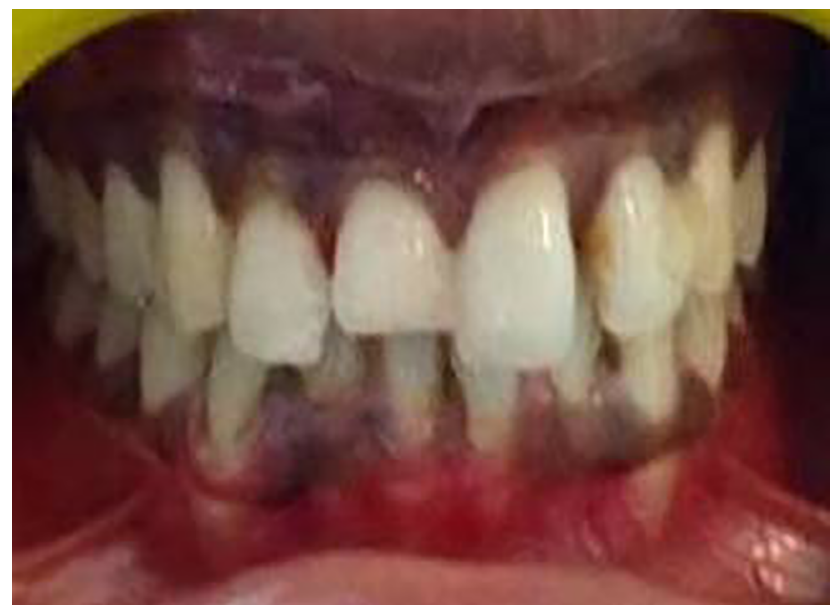

Fig. 2 Preoperative front view.

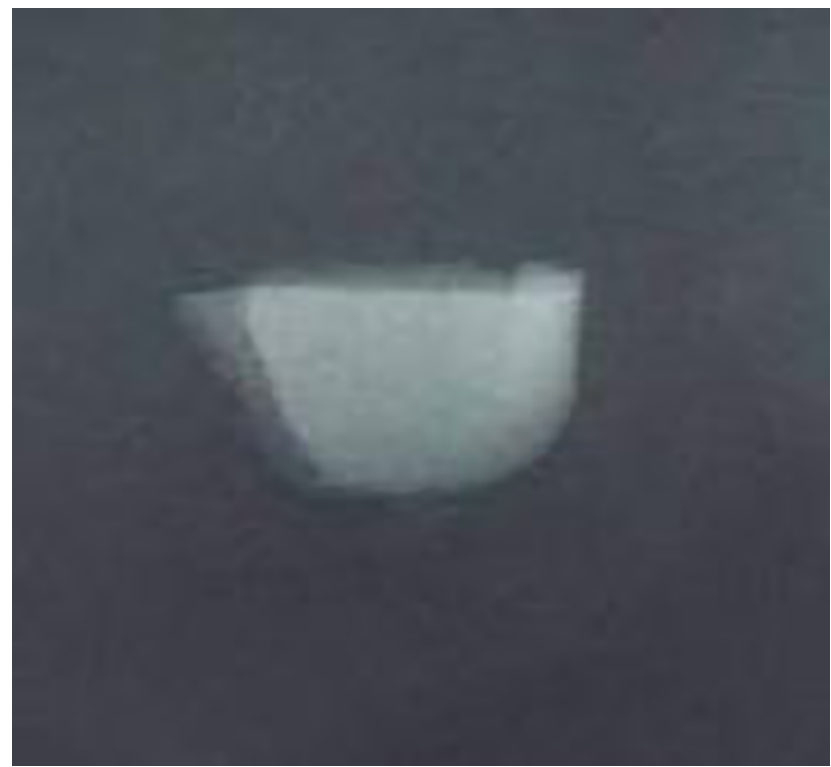

Fig. 4 Intraoral periapical radiograph of fractured tooth fragment.

1 week for the remaining treatment. Sutures were removed as the healing was satisfactory. The retrieved fractured maxillary central incisor fragment was then reattached with dual-cure composite resin (RelyX U200, 3M ESPE). Beveling of the tooth as well as of fractured segment was done, followed by acid etching for 20 seconds with $37 \%$ phosphoric acid. The etchant was thoroughly cleaned away for 10 seconds with a three-way syringe and the tooth was dried. Dual-cure composite resin adhesive system was adhered to the tooth fragment and repositioned to its proper position. As per the manufacturer's instructions, visible light polymerization curing was done while keeping the tooth fragment in position under finger pressure. Finishing and polishing was done with polishing discs and postinstructions were given. The patient was recalled for routine checkup after a week. No pathology was seen at the time of routine checkup. The patient as well as her parents was satisfied with the esthetic and outcome of the treatment. 


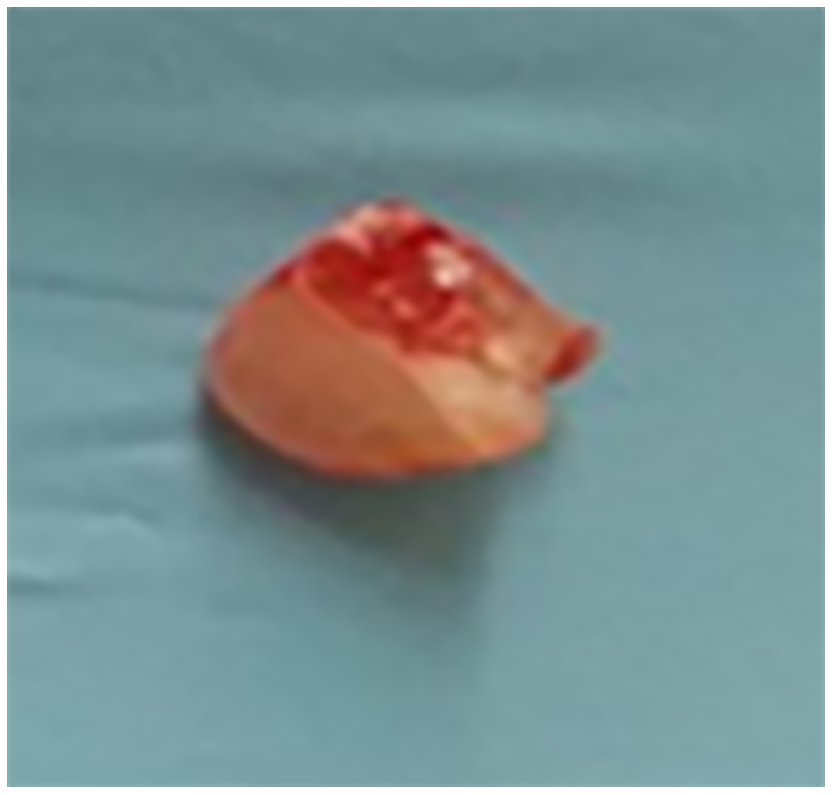

Fig. 5 Embedded tooth fragment.

\section{Discussion}

Dental injuries are frequent in children and adolescents, comprising $5.12 \%$ of all the injuries. ${ }^{7}$ Traumatic dental injuries occur mostly between age 2 to 3 years and 8 to 12 years. ${ }^{3}$ Soft-tissue laceration is commonly seen after the fracture of the incisors at the time of trauma. ${ }^{1}$ The incisal segment of broken tooth may penetrate in the full thickness of the lip causing lip laceration, if the impact of force is vertical and parallel to the long axis of the incisors. ${ }^{8}$ Attention should be paid for soft-tissue laceration for presence of fractured or missing tooth fragments. The fractured fragments may be lost, aspirated, swallowed, or can even get locked in the soft tissue, resulting in a serious hazard to the patient. ${ }^{4}$ Mostly the fractured fragments are left unnoticed by the primary health care workers during emergency management. Hence, a detailed case history, careful clinical examination of the laceration, and soft-tissue radiographs are mandatory to rule out any foreign object. If conventional radiographs are not enough, computed tomography (CT) or ultrasound can be used to rule out foreign objects in soft tissue. ${ }^{8}$ If the embedded tooth fragments remain undetected for a longer period of time, it may lead to infection, pain, swelling, scarring, fibrosis, and migration of the tooth fragment.

Differential diagnosis of embedded or locked tooth fragment includes soft-tissue calcification, foreign body, sialolith, and subcutaneous lesions. ${ }^{2}$ In the case of embedded fractured tooth fragment in the soft tissue, the best treatment choice is its retrieval by surgical excision. After the retrieval of the fractured fragment, a radiograph should be taken to ensure the complete removal of the fragment. If the fragment is not completely removed, it may cause tearing of the suture line, pus discharge, persistent chronic infection, and a disfiguring fibrosis. If the fractured fragment is intact, it should be reattached to the remaining fractured tooth. ${ }^{9}$

Fragment reattachment is a quick, low-cost, conservative technique that provides superior rehabilitation of

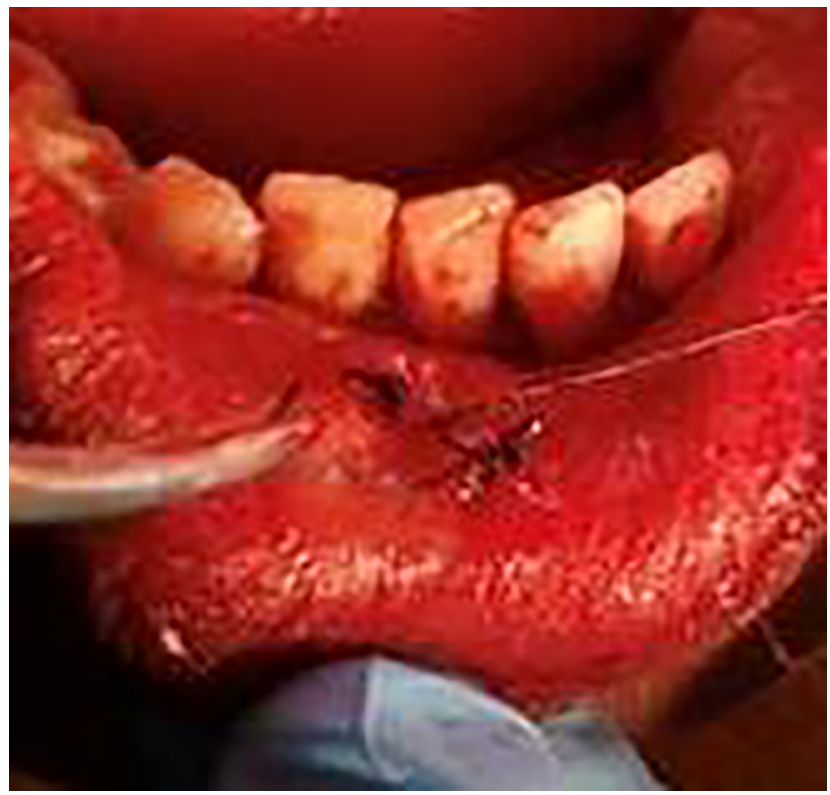

Fig. 6 Immediate postoperative intraoral view.

esthetics, function, and psychological support to the patient. The color-matching and incisal translucency are maintained and offer good reproduction of the shape, contour, and texture of the natural tooth. ${ }^{9,10}$ But the major drawback of the reattachment is inadequate rehydration of the fragment, leading to color change, fracture, and de-attachment of the fragment. 6

In this case report, it was observed that neither the medical practitioner nor the patient and her parents attempted to locate the tooth fragment at the site of injury. This case report emphasizes the need for clinical and radiographic examination of dental injuries with embedded fractured tooth segment in soft tissue of lower lip and its complete removal followed by reattachment.

\section{Conclusions}

The present case report accentuates the necessity for a detailed clinical and radiographic examination in each and every case of soft-tissue injuries that accompany a dental injury. An early diagnosis and the noncomplicated surgical removal of these fragments could prevent undesirable foreign body reactions and scarring. All attempts should be made to locate the missing tooth structure through a comprehensive history of any trauma and careful examination, so as to avoid any further complications.

\section{Conflict of Interest}

None declared.

\section{References}

1 Agarwal A, Rehani U, Rana V, Gambhir N. Tooth fragment embedded in the upper lip after dental trauma: a case report presenting an immediate diagnostic approach and complete rehabilitation. J Indian Soc Pedod Prev Dent 2013;31(1):52-55

2 Pandey R, Gupta R, Bhagat N. Tooth fragment embedded in lower lip following trauma. Int J Oral Health Sci 2017;7(2):105-107 
3 Marwaha M, Bansal K, Srivastava A, Maheshwari N. Surgical retrieval of tooth fragment from lower lip and reattachment after 6 months of trauma. Int J Clin Pediatr Dent 2015;8(2):145-148

4 Nagaveni NB, Umashankara KV. Tooth fragment embedded in the lower lip for 10 months following dentoalveolar trauma: a case report with literature review. Burns Trauma 2014;2(3):141-145

5 Simonsen RJ. Restoration of a fractured central incisor using original tooth fragment. J Am Dent Assoc 1982;105(4):646-648

6 Taguchi CM, Bernardon JK, Zimmermann G, Baratieri LN. Tooth fragment reattachment: a case report. Oper Dent 2015;40(3):227-234
7 David J, Astrøm AN, Wang NJ. Factors associated with traumatic dental injuries among 12-year-old schoolchildren in South India. Dent Traumatol 2009;25(5):500-505

8 Barua P, Chaudhary S, Kaur H, Mallikarjuna R. Treatment imprudence leading to missed tooth fragment. BMJ Case Rep 2013;2013:154-156

9 Sangwan S, Mathur S, Dutta S. Retrieval and reattachment of an elusive tooth fragment. J Indian Soc Pedod Prev Dent 2011;29(2):171-175

10 Manikandan S, Md Alam N. The hidden tooth: a case report. J Clin Diagn Res 2012;6(7):1350-1351 